\title{
Fauna und Flora im Pleistozän-Profil von Murg bei Säckingen und ihre Aussage zur Altersdatierung
}

\author{
Von Ekke W. Guenther und Fritz Tidelski, Kiel \\ Mit 4 Tabellen und 3 Abbildungen im Text
}

$\mathrm{Zus}$ ammenf assung: Das Profil von Murg zeigt über liegendem Gneis eine verlehmte Grundmoräne als unterste pleistozäne Schicht. Sie entstand während des weitesten Vorstoßes der Alpengletscher in Richtung zum Schwarzwald und wird seit PENCK \& BRÜCKNER (1909) in die Rißvereisung gestellt. Über ihr liegen pollenführende Ablagerungen eines Torfmoores und eines Teiches. Die Pollen weisen in den tieferen Lagen auf eine Pflanzengemeinschaft, die auch unter dem heutigen Klima noch möglich wäre. In den höheren Lagen verschwinden die wärmeliebenden Bäume. Das Gesamtdiagramm (Pollen-Spektrum) läßt die Zeitphase eines abklingenden Interglazials erkennen.

Uber diesen Sedimenten liegen 6-10 m Lösse und Lößlehme. Von äolischen Lössen, die je eine kalte Zeit vertreten, wird eine durchschnittlich $2,50 \mathrm{~m}$ mächtige Lehmlage eingerahmt, die mehr oder weniger deutliche Bodenbildungen enthält. Es läßt sich nachweisen, daß wenigstens ein Teil dieser Böden an Ort und Stelle entstanden ist. Da auch dieser Horizont eine warme Zeitphase repräsentiert, liegen im Profil von Murg über der Rißmoräne die Ablagerungen zweier Warmzeiten.

Reste von Elefant, Riesenhirsch und Bison erlauben eine Parallelisierung der "Murger“ Schichtfolge mit einem Teilabschnitt des Profils von Achenheim bei Straßburg. Hierbei zeigt es sich, daß die untere Warmzeit zwischen Moräne und unterem Löß den Schichten entspricht, die in Achenheim den Waldelefanten (Palaeoloxodon antiquns FaLC.) führen. Sehr wahrscheinlich sind diese gleichaltrig mit einem Teil der Travertine von Taubach, Weimar und Ehringsdorf. Der pollenführende Abschnitt von Murg wäre dann wohl in die Zeit der Bildung des oberen Travertins einzugliedern.

Die höher liegende von Lössen eingerahmte Lehmlage von Murg entspricht im Profil von Achenheim einem bis $5 \mathrm{~m}$ mächtigen Lehm zwischen Loess récent und Loess ancien supérieur. Nach Wernert (1957) gab es damals im Straßburger Gebiet offenen Wald und Steppe (Taiga). Es lebten Ren, Pferd, wollhaariges Nashorn und Mammut, aber auch Boviden, Edelhirsch und sogar Reh und Bär. Ein entsprechender Bodenhorizont konnte auch im Deckprofil der Travertine von Ehringsdorf nachgewiesen werden (GUENTHER 1958).

$\mathrm{Su} \mathrm{m}$ m a $\mathrm{y}$ : The Murg profile shows a loamy ground moraine as the lowermost Pleistocene layer over gneis. The moraine originated during the furthest thrust of the Alpine glaciers in the direction of the Black Forest, and has been placed since 1909 (according to the work of PENCK and BRÜCKNER) in the Riss glacial stage. Overlying the moraine are pollenbearing peat bog and pond deposits. The pollen indicate, in the deepest layers, a plant community that might still be possible under present climatic conditions. In the higher layers, the trees having affinities to warm conditions disappear. The composite diagram (pollen spectrum) indicates the end phase of an interglacial stage.

Over these sediments lie 6-10 m of loess and loess-loam deposits. A thick loam layer, averaging $2.5,0 \mathrm{~m}$ in width, which contains more or less distinct soil horizons, lies between aeolian loess, which always represents a cold period. It can thus be proved that at least a part of these substrata originated in place. Since this loam horizon also represents a warm phase, the deposits of two warm periods overlie the Riss moraine in the Murg profile.

Remains of elephants, giant stags, and bison permit comparison of the Murg stratigraphic sequence with a portion of the profile at Achenheim near Strassburg. Hereby is shown that merely the warm period, between the moraine formation and the lowest loess at Murg, can correspond to layers at Achenheim which have yielded the last ancient elephant (Palaeoloxodon antiquus FALC.). These are approximately of the same age as the travertine at Ehringsdorf. The pollen-bearing sectionat Murg might then probably be included in the time of formation of the upper travertine at Ehringsdorf.

\section{Einführung}

Die Vorbergzone des südlichen Oberrheintals, ein etwa 3-4 km breiter Streifen vordiluvialer Sedimente, begleitet auf weite Strecken den Westabfall des Schwarzwaldes und den Ostabfall der Vogesen. Fast immer sind die Vorberge von Löß überlagert. Auch der großenteils aus vulkanischen Gesteinen aufgebaute Kaiserstuhl in der Mitte des Rheintals trägt eine im Westen geringere, im Osten sehr mächtige Lößdecke. Auf das Grundgebirge 
greifen die echten Lösse nur selten über, und somit sind Vorbergzone und Kaiserstuhl im südlichen Oberrheintal die wichtigen Gebiete für die Lößforschung. Hier sind Lößüberlagerungen von mehr als $30 \mathrm{~m}$ erhalten.

Alle nicht zu geringmächtigen Lößprofile enthalten fossile Böden. Eine Zusammenstellung ergibt bisher 10 derartige fossile Klimazeugen, wobei einzelne Böden charakteristisch genug ausgebildet sind, um eine Parallelisierung auch bei größeren Abständen der Profile voneinander zu erlauben. Wichtige Profile, die bisher untersucht wurden, sind Riegel, Niederrotweil (Guenther 1961), Munzingen (prähistorische Fundstelle) und Heitersheim (wird zur Zeit in einer Inauguraldissertation von A. BRONGER analysiert). Hinzu kommen weiter im Norden bei Straßburg die Aufschlüsse bei Hangenbieten-Achenheim, die so zahlreiche Fossilien und Artefakte geliefert haben (WERnERT 1957).

Nur ein Teil dieser Profile erlaubt die Verknüpfung der Lösse mit Schottern, über deren Alter zudem noch zu diskutieren ist. Oberhalb von Basel fließt der Rhein in O-WRichtung in einer Senke zwischen dem Schweizer Jura und dem Schwarzwald. Auch hier kamen Lösse zur Ablagerung. Untersucht wurden bisher die Profile von Wyhlen bei Basel und von Murg etwa $4 \mathrm{~km}$ östlich von Säckingen.

In Murg besteht die Basis der Lösse aus Geschiebelehm, wodurch eine Verknüpfung dieses Profils mit den Vereisungen der Alpen und des Schwarzwaldes möglich ist. Die Moräne entstand während der bedeutendsten Vergletscherung dieses Gebietes, die eine Eisdecke von den Alpen bis über den Rhein vorschob und den Kontakt zu den Gletschern, die vom Schwarzwald herabflossen, herstellte. Das rißzeitliche Alter dieser alpinen Vereisung wurde schon früh erkannt (PENCK \& BRÜCKNER 1909), da sich ihre Schotterterrassen nach Höhenlage, Verwitterungsgrad und Lößbedeckung von den älteren Deckenschottern sowie der jüngeren Niederterrasse deutlich unterscheiden lassen. Die Verbindung zur Schwarzwaldvereisung konnte erst in neuerer Zeit hergestellt werden. In mehreren Veröffentlichungen haben Pfannenstiel und sein Mitarbeiter Rahm (PFannenstiel 1958, 1960, 1961, Pfannenstiel \& Rahm 1963, Reichelt 1955, 1960) vor allem an Hand von Geschiebeuntersuchungen den weitesten Vorstoß einer älteren Schwarzwaldvergletscherung aufgezeigt, und auf ihre Untersuchungen wird im folgenden zunächst Bezug genommen.

Die Schneegrenze des Schwarzwaldes lag damals bei $700 \mathrm{~m}$. Die morphologische Senke zwischen Schwarzwald und Schweizer Jura, in der auch Murg liegt, existierte schon. Sie wurde streckenweise von einem vielleicht $400 \mathrm{~m}$ mächtigen Eis plombiert. Die Schmelzwässer im Raum zwischen Waldshut und Säckingen mußten sich daher verschiedentlich unter dem Eis ihren Weg suchen. Wro Alpen- und Schwarzwaldgletscher zusammenstießen - es war dies im Albtal und dann wieder vom Schwarzatal nach Osten bis zur Wutach der Fall - bildeten sich mehrere $100 \mathrm{~m}$ breite Mischmoränen, die aus alpinen und aus Schwarzwaldgesteinen zusammengesetzt sind. Diese Mischzonen beweisen die Gleichaltrigkeit der beiden Eisvorstöße. Hierzu führen PFannenstiel \& RAHM weiter an: „Es kann auch kein anderer Gletscher einer anderen Vereisung die Geschiebe zurückgelassen haben als eben der Rißgletscher. Von den beiden ersten Vereisungen der Günz- und Mindelvergletscherung wissen wir gar nichts aus dem Schwarzwald. Wahrscheinlich waren der Schwarzwald und seine Ostabdachung im früheren Pleistozän nicht vereist,..."

\section{Der untere Abschnitt des Profils}

Das Gebiet von Murg liegt noch im Bereich der Alpenvergletscherung. Die Moräne enthält reichlich, große, kantengerundete Geschiebe. Es sind dies vor allem Quarzite, Granite, völlig verwitterte kristalline Schiefer und nur im Kieselskelett erhaltene „Kieselkalke". Die vielen kalkigen Bestandteile, die zunächst vorhanden waren, sind restlos gelöst und weggeführt worden (2. Diskordanz). Der ehemalige Geschiebemergel ist also zu Geschiebelehm verwittert, und diese Verwitterung hat auch den obersten Abschnitt des unterlagernden Gneises erfaßt. 
Da die hangenden Lösse - zum Teil sogar reichlich - Kalk enthalten, muß die Verwitterungsphase in die Zeit zwischen Eisbedeckung und Lößsedimentation eingegliedert werden. Im obersten Geschiebelehm fanden sich Strukturböden. Nach dem Rückschmelzen des Eises muß daher noch eine Zeit mit kalten Klimabedingungen berücksichtigt werden. Bereits Zink (1941) hatte auf dem Geschiebelehm Pflanzenreste beobachtet, die er jedoch für verschwemmt hielt. Die Oberfläche der Moräne ist uneben und fällt innerhalb der Grube generell nach Westen bzw. nach Nordwesten. In der tiefsten Senke fanden sich die Ablagerungen eines Torfmoores und eines Tümpels. Torfe und Seeablagerungen sind pollenhaltig, und eine durch Herrn Prof. TIDELski durchgeführte Pollenanalyse gibt einen Eindruck von der Pflanzengesellschaft und erlaubt damit einen Schluß auf die Klimabedingungen, unter denen diese Sedimente entstanden sind.

Der etwa 1,20 m mächtige pollenführende Profilabschnitt läßt sich von oben nach unten folgendermaßen untergliedern:

$10 \mathrm{~cm}$ helle, z. T. sandige Lagen mit einzelnen Torfbändern, etwas Kalk.

$40 \mathrm{~cm}$ Mehlsand, stark humos, mit zahlreichen Torfeinlagerungen (Torf-Gyttja). Kalkgehalt z. T. über $20 \%$.

$40 \mathrm{~cm}$ heller Torf, nur in den obersten $10 \mathrm{~cm}$ etwas Kalk, sonst kalkfrei.

$20 \mathrm{~cm}$ dunkler Torf, stärker zersetzt, kalkfrei, an der Basis Holzreste, die leider nicht geborgen werden konnten.

$5 \mathrm{~cm}$ Mehlsand, kalkfrei.

Darunter folgen zunächst feingeschichteter, verschwemmter Geschiebelehm und später nicht geschichteter Geschiebelehm.

Die Auflast von ca. $9 \mathrm{~m}$ Löß hat die Torflagen des Profils komprimiert. Die Mächtigkeit dieser Schicht muß vor der Ablagerung der Lösse weit größer gewesen sein.

Die Mehlsandlage (Gyttja) erinnert in Mineralzusammensetzung, Struktur und Textur an Sedimente des Spätglazials, wie sie in den eisrandnahen Gebieten NW-Deutschlands (Guenther 1960) zu finden sind. Hier wurde kleinen Teichen und Seen Material aus Geschiebemergeln zugeführt. Auch die dabei entstandenen Sedimente waren kalkreich.

Ohne Zweifel hatte die Rißmoräne vor ihrer Verwitterung einen nicht ganz geringen Kalkgehalt. Kalkteilchen wurden in Kornform verfrachtet. Zugleich wurde durch kohlensäurehaltige Niederschlags- und Grund-Wässer Kalk gelöst und aus dem Wassereinzugsbereich ebenfalls den Wasserbecken zugeführt, wo der Kalk durch biogene und abiogene Vorgänge wieder ausgefällt werden konnte. In den Zeiten eines wärmeren Klimas nimmt die physiologische Kalkausfällung zu, da durch den reicheren Bewuchs die Assimilationstätigkeit der Pflanzen und damit der Kohlensäureverbrauch vermehrt werden. Wächst der Humussäuregehalt jedoch zu stark an, bleibt der Kalk in Lösung. Im Torfsediment von Murg darf daher höchstens noch in den Grenzschichten etwas sekundär zugeführter Kalk erwartet werden.

\section{Der pollenanalytische Befund des unteren Profilabschnittes}

Zur pollenanalytischen Untersuchung gelangten aus der ca. 1,20 m mächtigen organogenen Sedimentfolge des unteren Profilabschnitts insgesamt 25 Proben. Die Aufbereitung des in bergfeuchtem Zustand entnommenen Materials für die Pollenanalyse erfolgte im Botanischen Institut der Universität Kiel mittels Flußsäure und nachfolgender Acetolyse. $\left.{ }^{\urcorner}\right)$ Ausgezählt wurden bei stark wechselnder Frequenz ie Präparat mindestens 100 Pollen. Die Pollenerhaltung war i. a. als gut zu bezeichnen. Die Darstellung (Abb. 1) erfolote in der herkömmlichen Form, bei der die Summe der Waldbaumpollen unter Ausschluß von Corylus die Bezugsgrundlage für alle übrigen Pollen- und Sporentypen abgibt.

1) Herrn Dr. AlEtsee sei an dieser Stelle für die Zurverfügungstellung des aufbereiteten Materials gedankt. 
Tabelle 1 Zählprotokoll der in Murg nachgewiesenen Pollen

\begin{tabular}{|c|c|c|c|c|c|c|c|c|c|c|c|c|c|c|c|c|c|c|c|c|c|c|c|c|c|c|}
\hline $\begin{array}{l}z \\
0 \\
0 \\
0 \\
0 \\
0 \\
0 \\
0 \\
0\end{array}$ & 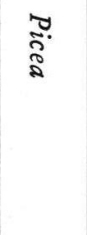 & ت્ & 寗 & $\begin{array}{l}\text { Do } \\
\text { इ } \\
\text { 章 }\end{array}$ & 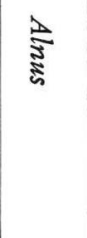 & : & $\underset{\Xi}{\Xi}$ &  & $\frac{\vec{d}}{\S}$ & $\begin{array}{c}M \\
\text { 囷 } \\
3\end{array}$ & 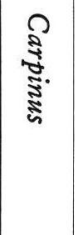 & $\frac{尺}{\stackrel{\$}{2}}$ & 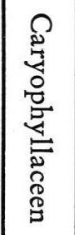 & 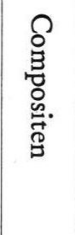 & 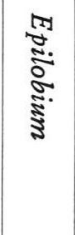 & 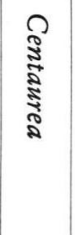 & 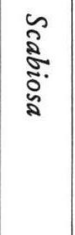 & 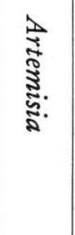 & 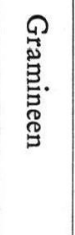 & 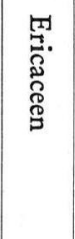 & 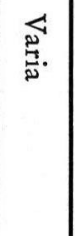 & $\begin{array}{l}M \\
Z \\
\text { 皃 }\end{array}$ & 武 & 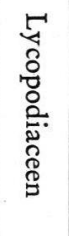 & 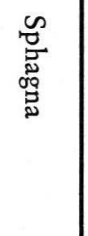 & 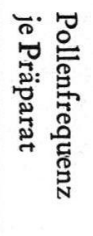 \\
\hline 0 & $\overline{5,17}$ & 75,86 & & 17,24 & 1,73 & & & & & & & & & 4,0 & & 1,0 & & 2,0 & 5,0 & & 3,0 & 15,0 & & & 3,0 & 66,6 \\
\hline 1 & 13,85 & $|73,85|$ & & 10,77 & 1,53 & & & & & & & & & $2,31 \mid$ & & & & & $0,77 \mid$ & & 0,77 & $3,85 \mid$ & & & 2,0 & 65 \\
\hline 2 & 35,0 & 52,0 & & 9,0 & 4,0 & & & & & & & & & & & & & & & & & & & & 2,0 & 17,6 \\
\hline 3 & 44,0 & 31,0 & & 14,0 & 11,0 & & & & & & & & & & & 1,0 & & & & & & 1,0 & & & 2,0 & 14,3 \\
\hline 4 & 64,44 & $|15,56|$ & & 18,52 & 1,48 & & & & & & & & & & & & & & & & 2,0 & 2,0 & & & 4,0 & 45 \\
\hline 5 & 75,73 & $|11,65|$ & & $2,91 \mid$ & $9,71 \mid$ & & & & & & & & & & & & & & & 1,0 & & 1,0 & 2,0 & & $2,91 \mid$ & 34,3 \\
\hline 6 & 81,49 & $8,77 \mid$ & & 2,92 & 6,49 & $0,33 \mid$ & & & & $0,33 \mid$ & & & $|\overline{0,33}|$ & & & & & & & & & $0,33 \mid$ & $|1,62|$ & 0,65 & $3,57 \mid$ & 154 \\
\hline 7 & $|81,17|$ & $|10,39|$ & & $4,54 \mid$ & 3,90 & & & & & & & & & & & & & & & & 1,95 & $1,95 \mid$ & $|0,65|$ & & $5,84 \mid$ & 154 \\
\hline 8 & 85,43 & 6,56 & 1,09 & 1,45 & $5,47 \mid$ & & & & & & & 0,36 & & 0,36 & & & & & & $|0,73|$ & & $1,09 \mid$ & $|1,09|$ & 1,09 & $0,73 \mid$ & 274 \\
\hline 9 & 76,23 & $|11,88|$ & $0,87 \mid$ & 3,48 & 7,25 & 0,29 & & & & $0,29 \mid$ & & 1,15 & & & 0,29 & & & & $0,29 \mid$ & & & $0,58 \mid$ & $|0,87|$ & & $3,48 \mid$ & 345 \\
\hline 10 & 60,0 & $|13,45|$ & 3,79 & 5,86 & 15,52 & 1,38 & & & & 1,38 & & 2,07 & & & & & & & & & $0,34 \mid$ & $0,34 \mid$ & & 1,03 & $14,83 \mid$ & 290 \\
\hline 11 & $\mid 66,28$ & $|14,53|$ & 2,04 & 4,36 & 12,21 & $0,58 \mid$ & & & & 0,581 & & 1,45 & & & & 0,29 & & & & & 0,29 & $0,58 \mid$ & $|0,29|$ & 0,58 & 6,10 & 344 \\
\hline 12 & 65,22 & 13,04 & 0,93 & 5,59 & 14,60 & 0,62 & & & & 0,62 & & 1,86 & & & & & & & & & & & $0,93 \mid$ & 0,31 & 12,11 & 322 \\
\hline 13 & 65,94 & $\mid 12,32$ & 3,62 & 3,44 & 13,59 & $0,73 \mid$ & & 0,36 & & 1,09 & & 1,81 & & & & & 0,18 & & & & & $0,18 \mid$ & $|1,81|$ & 0,18 & $8,51 \mid$ & 552 \\
\hline 14 & 64,74 & $|12,98|$ & $2,45 \mid$ & $4,74 \mid$ & 15,09 & & & & & & & 1,23 & & & & 0,18 & 0,35 & & & $0,18 \mid$ & 0,53 & $1,24 \mid$ & $|0,53|$ & & $4,21 \mid$ & 570 \\
\hline 15 & 67,81 & $|15,63|$ & 1,56 & $5,63 \mid$ & 9,06 & & & 0,31 & & $0,31 \mid$ & & & & & & $0,31 \mid$ & & $0,31 \mid$ & $0,31 \mid$ & & $0,31 \mid$ & $1,24 \mid$ & & & $8,13 \mid$ & 320 \\
\hline 16 & 69,78 & 13,48 & 1,33 & $6,67 \mid$ & 7,86 & $0,44 \mid$ & & 0,44 & & 0,881 & & 0,44 & $|0,30|$ & & & & & 0,30 & & $|0,30|$ & 0,44 & 1,34 & 0,89 & 0,89 & $16,74 \mid$ & 675 \\
\hline 17 & $|52,29|$ & $|20,61|$ & 3,05 & 9,54 & 14,13 & 0,38 & & & & 0,381 & & 0,76 & & & 0,38 & $0,76 \mid$ & & & & 0,38 & $2,67 \mid$ & $4,19 \mid$ & 1,15 & 0,38 & $35,50 \mid$ & 262 \\
\hline 18 & 59,23 & $|18,70|$ & 0,12 & 9,35 & 11,85 & & & $0,75 \mid$ & & $0,75 \mid$ & & 1,25 & $|0,25|$ & & & & $0,12 \mid$ & $0,37 \mid$ & & $|0,37|$ & 0,37 & $1,48 \mid$ & $|2,49|$ & & $27,68 \mid$ & 802 \\
\hline 19 & 42,09 & 4,82 & 0,45 & 2,55 & 48,27 & 1,0 & & $0,27 \mid$ & & $1,27 \mid$ & $|0,55|$ & 1,36 & & 0,09 & & & & & & & & 0,091 & 0,64 & 0,27 & $17,73 \mid$ & 1100 \\
\hline 20 & 46,47 & 4,32 & 0,17 & 3,64 & 41,0 & 1,74 & 0,17 & 1,08 & $0,17 \mid$ & 3,16 & $|1,24|$ & 5,73 & & & & & & $0,08 \mid$ & 0,081 & $0,08 \mid$ & 0,501 & $0,74 \mid$ & 0,66 & & $35,27 \mid$ & 1205 \\
\hline 21 & 70,65 & $9,51 \mid$ & 2,17 & 1,09 & 14,67 & 0,82 & & $0,54 \mid$ & & $1,36 \mid$ & $|0,55|$ & 8,70 & & & & & & & & & & & $|2,17|$ & & $28,53 \mid$ & 368 \\
\hline 22 & 61,87 & $|11,73|$ & 3,73 & 2,94 & 15,47 & 0,53 & & 3,20 & & 3,73 & 0,53 & 10,93 & & & & & & & & $|0,80|$ & $0,53 \mid$ & $1,33 \mid$ & $|5,60|$ & & $47,20 \mid$ & 375 \\
\hline 23 & 67,86 & $|11,79|$ & 1,78 & 2,86 & 12,50 & 1,07 & & $2,14 \mid$ & & $3,21 \mid$ & & 9,64 & & 0,36 & & & & & & & & $0,36 \mid$ & $|1,43|$ & & $60,71 \mid$ & 280 \\
\hline 24 & 64,94 & $|17,24|$ & 1,15 & 5,17 & 10,35 & $1,15 \mid$ & & & & 1,15 & & 2,30 & & 0,57 & & & & $0,57 \mid$ & & & & $1,14 \mid$ & $|0,57|$ & & $11,49 \mid$ & 174 \\
\hline 25 & $|49,17|$ & $|27,50|$ & & $8,33 \mid$ & 14,17 & & & & & & & 2,50 & & & & & & & & $|0,83|$ & $0,83 \mid$ & 1,66 & $|3,33|$ & & $9,17 \mid$ & 60 \\
\hline
\end{tabular}




\section{Der pollenanalytische Befund}

a) Die Baum-und Strauchvegetation

Das Diagramm zeigt eine deutliche Vorherrschaft der Nadelbäume (Picea und Pinus). Während der erste größere Profilausschnitt fast ausschließlich von der F i c h t e beherrscht wird, dominiert die K i e f e r von Probe $2 \mathrm{ab}$. Die Erlenkurve weist einen geschlossenen Verlauf auf, schwankt aber in ihren Werten zwischen ca. 1\% und 48\%. In Probe 20 wird Alnus subdominant und in der folgenden (19) übersteigt sie die Werte der Fichte.

Dieser Diagrammabschnitt zeigt bei Einschluß der Proben im Liegenden unter anderem eine auffallende Vertretung einer größeren Zahl von Waldkomponenten. Nachdem bereits in der ersten Probe (25) der analysierten Schichtfolge neben Fichte, Kiefer (letztere subdominant) und Erle auch die $\mathrm{H}$ as el auftritt, weist letztere in der Folgezeit ihren höchsten in Murg ermittelten Wert mit nahezu 11\% (Probe 22) auf, um dann stetig zurückzugehen. Nur in den unteren Lagen des stark humosen Mehlsandes (Gyttja) erreicht sie vor ihrem endgültigen Verschwinden aus dem Diagramm noch einmal einen Wert von über 2\%. Als weitere thermophile Elemente erscheinen neben der Hasel in diesem Diagrammabschnitt zunächst die Lin de, kurz darauf die E iche. Während erstere einen Höchstwert von 1,74\% erreicht, beträgt der der Eiche 3,20\% (Probe 22). An EMW-Komponenten treten auch $\mathrm{Ulme}$ und Ahorn auf. Ihr Nachweis bleibt jedoch nur auf Probe 20 beschränkt. Auch die T a n n e tritt bereits früh ins Diagramm ein. Ihre Werte schwanken im Bereich ihres Vorkommens (Proben 24-8) zwischen 0,2\% und fast 4\%. Der bezeichnende untere Teil des Diagramms ist ferner durch das Vorkommen der $\mathrm{Ha}$ in $\mathrm{b} \mathrm{u} \mathrm{ch}$ e charakterisiert. Die Frequenz ist allerdings sehr niedrig. Als Maximalwert wurden 1,24\% ermittelt. In der ganzen Folgezeit ließ sich Carpinus nicht mehr nachweisen.

Der Profilausschnitt unter- und oberhalb des Erlenmaximums ist also durch eine Anzahl anspruchsvollerer Waldvertreter - jedoch mit geringen Prozentwerten - gekennzeichnet. Das Waldbild zeigt in diesen Diagrammausschnitten seine größte Mannigfaltigkeit. Hinweise auf eine dichte Bewaldung geben neben der geringen NBP-Beteiligung die

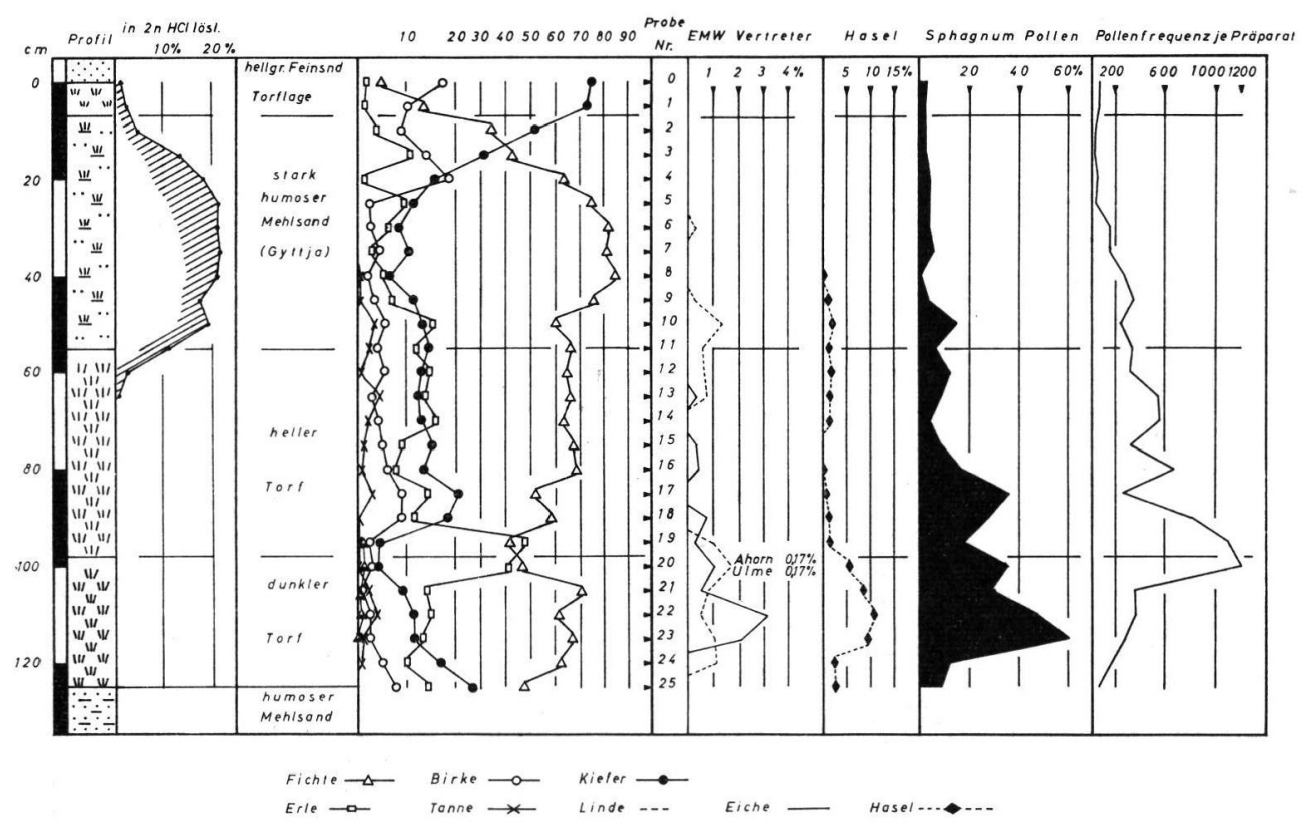

Abb. 1. Der Pollen-führende Abschnitt des Profils von Murg. 
absoluten Pollenfrequenzen, die in Probe 201205 und in Probe 19 noch 1100 je Präparat aufweisen.

Eine deutliche Verarmung an bestandsbildenden Waldvertretern läßt sich von Probe 7 ab feststellen. Aus den Spektren verschwinden die Komponenten des Eichenmischwaldes ganz. Nur in Probe 6 tritt bei 308 ausgezählten Pollen noch 1 Lindenpollenkorn auf. Auch die Tanne und die Hasel verschwinden. Die Kurve der Erle ist, abgesehen von 2 kleinen Anstiegen in diesem Profilabschnitt, rückläufig. Sie sinkt bis auf knapp 2\% (Proben 4, 1, 0). Die Fichte - im unteren und mittleren Abschnitt noch eindeutig dominierend - zeigt von Probe 6 ab einen kontinuierlichen Abfall von 80\% auf ca. 5\%. Der abschließende Diagrammabschnitt ist charakterisiert durch die Dominanz der Kiefer und einen Birkenanstieg.

b) Die Nichtbaumpollen

Auffällig ist für das ganze Diagramm der geringe Anteil an Nichtbaumpollen. Nachgewiesen werden konnten: Caryophyllaceen, Compositen, Epilobium, Centaurea, Scabiosa, Artemisia, Gramineen und Ericaceen (siehe Tabelle). Die höchsten Werte erreichen die NBP erst in der Kiefernzeit. Regelmäßig sind zudem Sphagnum-Sporen vertreten. Sie erreichen ihre größte Häufigkeit im Abschnitt, der durch die Anwesenheit der EMW-Vertreter, Carpinus und Corylus gekennzeichnet ist. Der ermittelte Maximalwert liegt bei gut $60 \%$ im Bereich des dunklen Torfes. Thre hier häufige Vertretung weist auf eine starke Vernässung hin.

c) Zur Frage der Buchenvertretung

Bei einer Übersichtsuntersuchung fand ALETSEE (nach freundlicher mündlicher Mitteilung) in unteren Bereich des dunklen Torfes (? Probe 23) ein gut bestimmbares Pollenkorn von Fagus. Da die Anwesenheit der Buche für die zeitliche Zuordnung der Schichtfolge von Murg von Wichtigkeit ist, wurde dieser Befund bei der Analyse der gesamten Probenserie besonders berücksichtigt. Trotz eingehender Durchsicht gelang es mir aber nicht, Buchenpollen festzustellen. Es kann sich also bei der Bestimmung durch Aletsee nur um den Nachweis eines vereinzelt gefundenen Buchenpollenkornes handeln. Von vereinzelten Buchenpollen berichtet auch LüDI (1958) aus dem schweizerischen Alpenvorland. Sie gehören nach seinen Untersuchungen jüngeren, kiefern- und fichtenbeherrschten Interglazialabschnitten an. Auch ReIch (1953) erwähnt ein vereinzeltes Pollenkorn von Fagus im Profil von Großweil III. Averdieck (1962), der eine Neuuntersuchung des Profils von Fahrenkrug/Holstein durchführte, fand bei seinen Untersuchungen keine Spur der Gattung Fagus. Fahrenkrug gehört dem letzten Interglazial (Saale/Warthe-Weichsel-Interglazial) an. In Odderade in Dithmarschen gelang es AvERDIEck jedoch, auch ein vereinzeltes FagusPollenkorn nachzuweisen. Er stellt diese Lokalität ins Brørup-Interstadial. Das völlige Fehlen bzw. das vereinzelt gefundene Pollenkorn der Buche - sicher auf Fernverwehung zurückzuführen - gäbe einen Hinweis dafür, daß das Diagramm von Murg jenen Diagrammen zuzuweisen ist, die durch das $\mathrm{Fehlen}$ von $F$ agus charakterisiert sind und damit ein interglaziales Alter aufweisen. Mit Sicherheit tritt in Murg die Hainbuche an Stelle der Buche.

d) Zur Frage der Picea-und Pinusarten

Bei der Durchsicht der einzelnen Proben fiel auf, daß besonders die oberen Diagrammabschnitte neben der Hauptmasse an großen Fichtenpollen auch durch das Vorkommen kleinerer Piceapollen gekennzeichnet sind. Durchgeführte Messungen ergaben für die großen Fichtenpollen Längenwerte zwischen 90-140 $\mu$. In Ausnahmefällen stieg der Wert bis $150 \mu$. Die gemessenen Werte der Luftsackhöhen liegen zwischen 50-80 $\mu$, im Mittel bei $71 \mu$. Die kleineren Fichtenpollen maßen in der Länge $60-70 \mu$, die Luftsackhöhen lagen im Mittel bei 52,5 $\mu$. Sie fallen damit in den Bereich kleinerer Pollen vom OmorikaTyp. Dieser Nachweis erlaubt den Schluß, daß diese Fichtenart an der Zusammensetzung 
der Nadelwaldbestände in der Schlußphase der limnischen Sedimentation in Murg beteiligt war.

In Probe $O$ (Kiefernzeit) wurden auch Messungen an Kiefernpollen durchgeführt. Die I.änge derselben schwankt zwischen 50-80 $\mu$. Während die kleineren Typen (unter $65 \mu$ ) auf Pinus silvestris hinweisen, sind die größeren (zwischen $65-80 \mu$ ) Pinus mugo (nach Lüni 1958, S. 30) zuzuweisen. Damit ist für diese Probe auch der pollenanalytische Nachweis der Bergkiefer wahrscheinlich gemacht.

So repräsentieren die oberen Spektren ein Waldbild, das sich aus Vertretern beider Föhrenarten zusammensetzt. Daß das Waldkleid noch dicht war, läßt sich durch die geringen NBP-Werte (maximal 15\%) erweisen.

\section{Zeitliche und klimatische Stellung der Schichtfolgen}

Nach den Befunden erweist sich das vorgelegte Diagramm als interglazial. Das Vorherrschen der Fichte und Kiefer deckt sich durchaus mit den Befunden LüDI’s (1953 u. 1958) aus dem schweizerischen Alpenvorland. Der untere und mittlere Teil (bis einschließlich Probe 8) mit seinen geringen Anteilen an wärmeliebenden Vertretern (Hasel, Eiche, Linde, Ulme, Ahorn) stellt das Diagramm von Murg in den End-Abschnitt der Vegetationsentwicklung eines Interglazials. Anzeichen für ein ausklingendes Optimum treten in den Proben 19 und 20 auf; nicht nur die hohe Pollenfrequenz, auch die relative Häufung wärmeliebender Vertreter deuten darauf hin. Der hohe Anteil von Sphagnumsporen im liegenden Teil des analysierten Profils weist auf ein sphagnumreiches Moor hin. Der Maximalanstieg der Fichte auf ca. $80 \%$ in den Proben 6-8 läuft parallel mit dem Verschwinden der letzten Vertreter einer wärmeliebenden Flora. Von Probe 6 ab beginnt der kontinuierliche Anstieg der Kiefer, in den letzten Spektren auf nahezu 76\%. Nicht nur der Kieferanstieg, sondern auch der der Birke auf fast $18 \%$ deuten auf eine Klimaverschlechterung im Ausgang der erfaßten Vegetationsentwicklung hin. Die hohen Werte von Alnus in Probe 19 sind sicher lokal bedingt, wenn auch die durchlaufende Kurve der Erle auf ein regelmäßiges Vorkommen im Bereich von Murg hinweist. Der Gesamtbefund mit den ausgesprochenen Picea- und Pinuswerten zeigt viele Analogien zu den Befunden von LüDI und REICH im schweizerischen und bayerischen Alpenvorland.

Der Klimacharakter weist für den gesamten pollenanalytisch untersuchten Ablagerungskomplex eindeutig auf eine gemäßigte kontinentale Färbung hin. Sie ist bezeichnend für das ausklingende Riß/Würm-Interglazial, das nach FIRBAS (1958, S. 87) durch keine größeren Kälteschwankungen, insgesamt gesehen, unterbrochen ist. Diese Feststellung läßt sich auch durch unsere Untersuchungsbefunde erhärten. Die stratigraphischen Verhältnisse (s. Abschnitt 1) bestätigen die zeitliche Einstufung der Torf- und Gyttjaablagerungen von Murg.

\section{Ergebn is}

Das Diagramm von Murg zeigt eine ausgesprochene Dominanz der Nadelhölzer. Es ist deutlich zweigeteilt. Ein langer älterer Abschnitt (Proben 8-25) ist gekennzeichnet durch die Vorherrschaft der Fichte. Wärmeliebende Waldkomponenten - mit sehr geringen Werten vertreten - charakterisieren in geschlossener Kurve nur die liegenden Sedimentfolgen. In Probe 6 gelang der letzte Nachweis der Linde. Mit dem Abstieg der Fichtenkurve korrespondiert ein kontinuierlicher Kiefernanstieg mit Höchstwerten in den letzten Proben des Diagramms.

Ein Klimaoptimum dokumentiert sich in den Proben 19 und 20. Zu diesem Zeitpunkt gewinnt vorübergehend die Erle die Führung im Diagramm. Diese Dominanz ist sicher lokal bedingt.

Der Gesamtcharakter der Vegetationsentwicklung im Profil von Murg weist unter Berücksichtigung der Baum- und Strauchvertreter wie der Nichtbaumpollen auf einen EndAbschnitt eines Interglazials mit einem geschlossenen Waldkleid hin. 


\section{Der Klimaablauf der Zeit der Bildung des unteren Profilabschnittes}

Die Auswertung der Untersuchungen des unteren Abschnitts des Murger Profils zeigt damit folgende Entstehungsgeschichte:

Eine mächtige Lage von Gletschereis, das, aus dem Alpengebiet kommend, nach Norden vorstieß, füllte teilweise die Senke zwischen Schweizer Jura und Schwarzwald aus und überfuhr etwa $2 \mathrm{~km}$ über Murg hinaus den Südfuß des Schwarzwaldes. Aus einer späteren immer noch kalten Phase, als das Eis schon wieder zurückgeschmolzen war, sind Strukturböden erhalten. Es folgte ein vielleicht längerer Zeitabschnitt, von dem die Überlieferung fehlt. Vielleicht haben wir es mit einer Unterbrechung der Sedimentation zu tun, vielleicht ist bereits abgelagertes Gestein später wieder abgetragen worden.

Irgendwann wurde es dann erheblich wärmer. Wahrscheinlich erst aus der Zeit nach dem Maximum der warmen Phase sind wieder Sedimente erhalten. Es bildete sich Torf. Im Pollendiagramm sind Linde, Eiche, Ahorn, Ulme und Hasel sporadisch in Teilkurven vertreten. Das sehr starke Vorherrschen von Fichte und Erle kann für ein feuchtes Klima sprechen. Noch fehlen zunächst Spätfröste und scharfe Winterfröste. Erst im oberen Teil des pollenführenden Abschnitts verschwinden die Baumarten, die ein wärmeres Klima beanspruchen, und zuletzt dominieren Kiefer und Birke. Eine neue kalte Phase beginnt.

Nur die untere Hälfte dieser Serie besteht aus rein phytogenen Sedimenten. Die obere Hälfte, wo es, nach den Pollen zu urteilen, bereits kälter wurde, enthält die Gyttja-artigen Ablagerungen eines eutrophen Gewässers. Feingeschichtete, kalkreiche Mehlsande voller Pflanzenreste wurden sedimentiert. Faust- bis kindskopfgroße Gerölle im obersten Abschnitt könnten durch treibende Eisschollen, vielleicht aber auch durch menschliche Einwirkung in das feinkörnige Sediment gekommen sein.

\section{Der obere, aus Lössen bestehende Teil des Profils}

Die pollenführende Serie wird von etwa $9 \mathrm{~m}$ Löß überlagert. Die neu hereinbrechende Kaltzeit begünstigte bei schütterer Vegetation die Verwehung von Lößstaub. In den durch Niederschlag verschwemmten Grenzschichten von Mehlsand und unterem Löß fand E. SCHMID (1949) Krotowine, die ausgefüllten Gänge höhlenbauender Nagetiere. Später folgten dann in der äolischen Ablagerungsform noch erhaltene Lösse, also Zeugen eines kalten, vielleicht trockenen Klimas. Diese Abfolge: Torfmoor - gvttjenbildender SeeNiederschlagseinfluß - äolischer Löß zeigt die umgekehrte Reihenfolge einer Sedimentation, wie sie aus der Übergangszeit vom Würmglazial über das Spätglazial zum Holozän vorkommt, umgekehrt deswegen, weil das Murger Profil eine Klimaentwicklung von Warm zu Kalt zeigt.

Uber etwa $2 \mathrm{~m}$ unterem Löß folgt eine Bodenbildung, die das Gestein zu einer Parabraunerde umwandelte. Diese Bodenbildung ließ sich mit einer Mächtigkeit um $\pm 2.50 \mathrm{~m}$ durch die ganze Grube (über $100 \mathrm{~m}$ ) verfolgen. Die Schicht ist entkalkt. Ein wesentlicher Teil des Bodenmaterials ist, wie die Feinschichtung zeigt. durch Niederschlagswasser verlagert worden, und man muß fragen. ob es sich nicht vielleicht bei der ganzen Lage um einen verschwemmten Horizont handelt. Nach der Morphologie ist für den größten Teil der Grube eine Verschwemmung lediglich vom Kalvarienberg her möglich. Seine höchste Erhebung, wo heute Gneis zu Tage tritt, liegt ca. $30 \mathrm{~m}$ südwestlich der Ziegeleiwand. Damit fehlt eine Fläche, die groß genug wäre, um eine so große Menge von Lehm, wie sie durch die Ziegeleigrube aufgeschlossen wurde, verwittern zu lassen. Die Verlagerung kann daher stets nur wenige Meter betragen haben.

Weitere Beobachtungen sprechen dafür, daß auch Verwitterung an Ort und Stelle stattfand. Im Löß unterhalb des entkalkten Lehms findet sich eine Zone der Kalkanreicherung mit beginnender Lößkindelbildung. Ferner sind in einzelnen Lagen die Reste von Kotballen von Würmern erhalten, die auch bei geringfügiger Umlagerung zerstört worden 
wären. An einigen Stellen konnte eine schwache „Pseudomycel“-Bildung beobachtet werden. Hierbei handelt es sich nicht um die feinen Kalkfäserchen, die in bodenkundlichen Arbeiten oft als „Pseudomycel“ bezeichnet werden und die sich bei sekundärer Kalkverlagerung in Schichten bestimmter Dichte und Plastizität bilden. Vielmehr ist in Murg ein fossiles, feinstfaseriges, phytogenes Zellgewebe, das nur bei stärkerer Vergrößerung sichtbar wird, erhalten. Entsprechendes ließ sich bisher nur in den Schichten einer kräftigen Bodenbildung nachweisen.

Alle diese Beobachtungen führen zu dem Schluß, daß die $\pm 2,50 \mathrm{~m}$ mächtige Lehmschicht der Zeuge einer intensiven Bodenbildung ist, wie sie nur bei einem wärmeren Klima entstehen konnte. Dabei zeigt das Lagerungsgefüge, daß Niederschlagswasser bei der Entstehung der Schicht eine wesentliche Rolle spielte. Vor allem in den oberen Abschnitten des Bodens ist Umlagerung besonders deutlich. Nach großenteils verschwemmten Schichten folgt nochmals ein geringmächtiger verbraunter Horizont, der dann von größtenteils äolisch sedimentiertem Löß überlagert wird. Die Vegetation fehlte, es war wieder kalt geworden.

Im jüngsten Abschnitt des Profils liegen über diesem Löß wieder verschwemmte Schichten. Zu unterst findet sich eine schwache Bodenbildung, darüber folgt durch Niederschlagswasser umgelagertes Sediment, das nach oben in den holozänen Boden übergeht. Dieser Boden wird an mehreren Stellen von entkalkten, verschwemmten Schichten überlagert. Als Folge des Ackerbaus fehlte zu bestimmten Jahreszeiten die schützende Vegetation, und so konnte der Niederschlag in jüngster geschichtlicher Zeit wieder Lößmaterial verlagern.

Die Untersuchung des Murger Profils zeigt demnach, daß nach der großen Vergletscherung, die das Alpeneis bis zum Südfuß des Schwarzwaldes vorstoßen ließ, vor dem Holozän 2 bedeutende warme Zeitabschnitte lagen, die von Kaltzeiten eingerahmt wurden. Das Klima der älteren Warmzeit war nach den Ergebnissen der Pollenanalyse kaum wesentlich kälter als das der Gegenwart, wobei offenbleiben muß, ob nicht ein noch wärmerer Zeitabschnitt direkt voranging, von dem die Überlieferung fehlt. Über das Bildungsklima des zweiten Bodens wissen wir weit weniger. Wenn man jedoch die bei der bodenkundlichen Lößstratigraphie üblichen Schlußfolgerungen zieht, muß auch in der Zeit der Entstehung dieser Schicht ein warmes Klima geherrscht haben.

\section{Diskordanzen im Profil}

Unterbrechungen in der Sedimentation und Erosionsdiskordanzen sind für die Ausdeutung eines Profils von entscheidender Bedeutung. Liegen sie innerhalb von Lössen, sind sie makroskopisch meist nicht und mikroskopisch oft nur schwer zu erkennen. Auf dem Diagramm (Abb. 2) sind ganz links neben der Schichtfolge die im Profil gut erkennbaren Diskordanzen in einer eigenen Rubrik angegeben. Sie finden sich (von unten nach oben) in folgenden Schichtabschnitten:

1. An der Oberfläche des Gneises: Die Abtragung von mehr als $700 \mathrm{~m}$ permischer, mesozoischer und ältestkänozoischer Sedimente fällt wohl zum größten Teil in die Zeit des oberen Tertiärs. Die Abtragung älterer pleistozäner Schichten kann bis kurz vor der Vergletscherung des Gebietes gegangen sein.

2. An der Oberfläche des Geschiebelehms: Dieser ist tiefgründig verwittert. Der ältere Abschnitt der warmen Zeitphase, die diese Verwitterung verursachte, ist nicht mehr vorhanden.

3. Im Bereich der verschwemmten Schichten vor Beginn der Lößablagerung.

4.-6. zu Beginn, innerhalb und am Ende des entkalkten Abschnittes. Zwischen 3,50 und 7,00 m Tiefe liegen mehrere Umlagerungsphasen, die in verschiedenen Profilabschnitten jeweils andere Schichten erfassen. 
7. Nach Ablagerung des oberen äolischen Lösses: Wahrscheinlich fehlen hier recht erhebliche Lößmengen. Das Profil zeigt somit nur kurze Zeitabschnitte, aus denen Sediment erhalten ist, neben langen Zeiträumen, aus denen die Gesteinsüberlieferung fehlt.

Die Lückenhaftigkeit, die jedes größere Profil terrestrischer Sedimente besitzt, kommt bei der Profiluntersuchung von Murg besonders deutlich zum Ausdruck und läßt sich mit Hilfe der besonderen Untersuchungsmethoden auch aufzeigen.

\section{Funde fossiler Säugerreste in der Ziegeleigrube von Murg}

Bereits ZINK (1940) gibt eine Zusammenstellung der Fossilfunde von Murg. Demnach stammen aus dem älteren Löß: Mammutbus primigenius, Rangifer tarandus, Coelodonta antiquitatis und Bos primigenius. Bei weiteren Funden ist ungewiß, ob sie aus dem älteren oder dem jüngeren Löß stammen. Es sind dies: Coelodonta antiquitatis, Bison priscus, Bos primigenius, Cervus elaphus, Cervus alces, Equus caballus. Durch Funde, die von Herrn E. Gersbach geborgen wurden und deren Lager fixiert werden konnte, ist diese Liste zu ergänzen.

B is on. Aus der hellen Schicht dicht über dem Torflager stammt der Atlaswirbel eines Bisons. Seine Maße wurden bereits in einer Liste, in der Atlaswirbel von Boviden und Cerviden verglichen wurden, veröffentlicht (Guenther 1962). Danach unterscheidet sich der Murger Atlas durch seine geringe Größe und durch bestimmte Verhältniszahlen von allen anderen, besonders jüngeren, untersuchten Bisonten. Die Maße der Tabelle mögen dies erläutern.

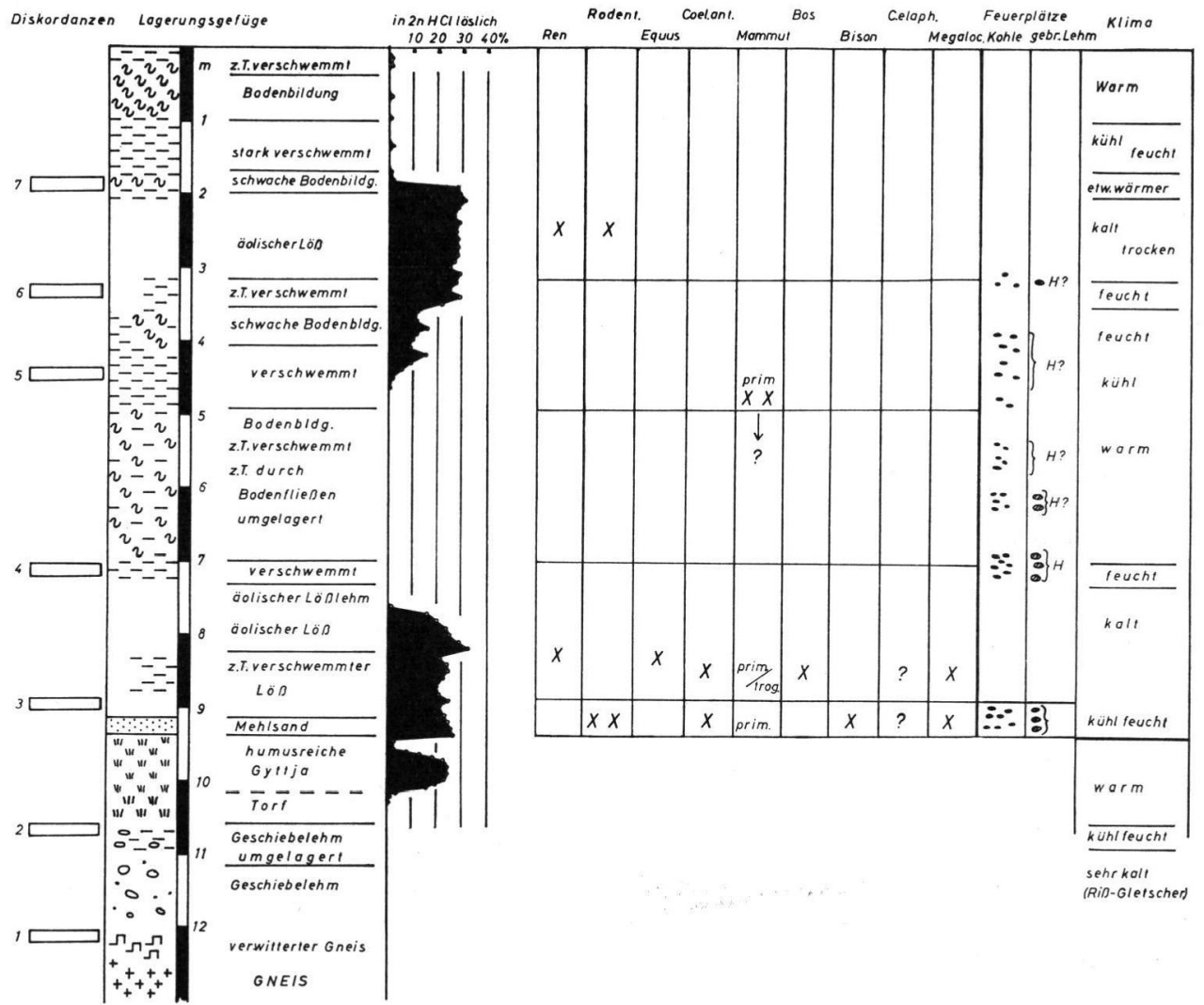

Abb. 2. Schichtfolge und Fauna des Profils von Murg. 
Tabelle 2

Maße des Atlas-Wirbels (in mm) von verschiedenen Bisonten

\begin{tabular}{lccc}
\hline & $\begin{array}{c}\text { Atlas d. Murger } \\
\text { Bisons }\end{array}$ & $\begin{array}{c}\text { Mittlerer Wert } \\
\text { von 4 } \begin{array}{c}\text { würmzeitlichen } \\
\text { Bisonten }\end{array}\end{array}$ & $\begin{array}{c}\text { Mittlerer Wert } \\
\text { von 4 rezenten } \\
\text { Wisenten }\end{array}$ \\
\hline Flügelbreite & 160 & 245 & 161 \\
Flügellänge & 87 & 151 & 110 \\
Länge des Arcus dors. & 50 & 74 & 55 \\
Vordere Gelenkfläche & $105 \times 50$ & $142 \times 66$ & $112 \times 56$ \\
Foramen vertebrae (Caudale Seite) & 40 & 64 & 46
\end{tabular}

Aus der Zusammenstellung ist zu ersehen, daß der Atlas-Wirbel des Bisons von Murg in allen Maßen wesentlich kleiner ist als die entsprechenden Wirbel der vor allem würmzeitlichen Bisonten. In der Breite der Alae erreicht er gerade den rezenten Bison europaeus Ow., bleibt aber schon in der Flügellänge weit hinter diesem zurück. Geschlechtsdiamorphismus kann hierbei nur eine unwesentliche Rolle spielen, da von den 4 Atlas-Wirbeln des Bison europaeus wenigstens 3 von weiblichen Tieren stammen. Wenn der Schluß von den Ausmaßen des Atlas-Wirbels auf die Größe statthaft ist, gehörte der Bison von Murg $\mathrm{zu}$ einer kleineren Art.

Von vielen Palaeontologen werden 2 Entwicklungslinien der pleistozänen Bisonten unterschieden. Es sind dies die Linie eines großen Steppenbisons und diejenige eines kleinen Waldwisents. Der erstere wird dem Bison priscus Boj. und der letztere dem Bison europaeus Ow. (=B. bonasus L.) gleichgestellt. Beide Formen scheinen vom frühen Pleistozän an nebeneinander gelebt zu haben. Die kleine Form wurde von Schoetensack in den Sanden von Mauer beobachtet, von Freudenberg dann genauer untersucht und als Vorfahre des Bison bonasus, der in geringen Resten heute noch lebenden Waldform, angesehen. Wernert (1957) konnte die Art an Hand von Fußknochen im „Löß ancien moyen“ von Achenheim bei Straßburg nachweisen.

Die Zugliederung des Murger Atlaswirbels zu diesen Formen bereitet Schwierigkeiten. Zum Steppenbison (Bison priscus BoJ.) kann er keinesfalls gestellt werden. Die Unterschiede sind zu groß. Aber auch von Bison europaeus Ow. (= bonasus L.) trennen ihn mehrere Merkmale, so die Flügellänge, die Länge des Arcus dorsalis, der Abstand der vorspringenden Enden der Gelenkflächen und vor allem die Ausbildung des Foramen vertebrae (Guenther 1962).

$\mathrm{R}$ iesenhirsch. Eine Bereicherung der Aufsammlungen in Murg bedeuten die Reste zweier Unterkiefer von Riesenhirschen (Megaloceros giganteus ALDR.). Ein etwas besser erhaltenes Stück stammt nach Mitteilung von Herrn E. GERSBACH sen. aus dem unteren Löß. Der Erhaltungszustand bestätigt ein kalkreiches Sediment. Im Mandibelkanal waren noch Lößreste vorhanden, deren Kalkgehalt bei 24\% lag. Das Stück ist auf der lingualen Seite zerbrochen, vielleicht durch Hyänenverbiß. An Zähnen sind erhalten $\mathrm{M}_{1}$ bis $\mathrm{M}_{3}$. Die Wurzeln des $\mathrm{P}_{3}$ und $\mathrm{P}_{4}$ stecken noch im Kiefer. Nach dem Abkauungszustand der Zähne handelt es sich um ein nicht mehr junges Tier. Das Stück zeichnet sich durch eine dunkelbraune Farbe aus.

Herr Dr. Wernert aus Straßburg, dem ich den Kiefer zum Vergleich mit entsprechenden fossilen Resten vorlegte, teilte mir mit: „Verglichen mit allen Megaceros-Mandibeln von Achenheim zeichnet sich die aus Murg durch folgende Charakteristika aus, wobei sich vorwegnehmen läßt, daß sich ein Vergleich mit dem Material aus älterem temperiertem Löß erübrigt, da dieser in jeder Hinsicht graziler ist. Der Kiefer zeigt eine außerordentliche Dicke unter dem $\mathrm{M}_{3}$ ( sie ist durch Quetschung noch besonders vergrößert). Dieses Maß nimmt verhältnismäßig langsam in Richtung auf das Diastema bis unter $\mathrm{P}_{4}$ und $\mathrm{P}_{3}$ ab. Der $\mathrm{M}_{3}$ ist durch Länge und Breite bemerkenswert. Ein verdoppelter accessorischer Pfeiler 
ist zwischen dem mittleren und dem hinteren Lobus der labialen Schmelzwand des $\mathrm{M}_{3}$ ausgebildet und ein einfacher Pfeiler zwischen dem vorderen und dem mittleren Lobus."

Der erstere accessorische Pfeiler fehlt bei allen Unterkiefern von Megaloceros von Achenheim, sowie den Mandibeln, die in den Paläontologischen Museen von Berlin und Wien untersucht werden konnten.

Zur diagnostischen Auswertung der auffallenden Dicke der Mandibel wurde von allen vermessenen Unterkiefern ein Quotient errechnet, der die Höhe zur Breite in Beziehung setzt. Sie ergibt sich aus: mittl. Wert der Höhe d. lingualen und buccalen Seite unter $M_{1}$, $\mathrm{M}_{2}, \mathrm{M}_{3}$ dividiert durch die Dicke des Kiefers unter $\mathrm{M}_{1}, \mathrm{M}_{2}, \mathrm{M}_{3}$. Die gefundenen Quotienten wurden nach dem geologischen Alter der fossilen Kiefer geordnet. Als Ergebnis zeigte sich - innerhalb einer nicht unbeträchtlichen Variation - eine Abnahme der relativen Dicke während der Würmvereisung. Der außerordentlich kräftige Unterkiefer von Murg paßt hiernach am besten zu Funden von Phöben bei Potsdam, einer Fundstelle, die nach Wolnstedt (1958) dem letzten Interglazial zuzugliedern ist.

Zur Frage der Parallelisierung mit Achenheimer Funden käme wohl am ehesten das Fragment eines linken Mandibelastes in Betracht, der mehr oder weniger dem Murger Stück entsprechen könnte (gefunden am 8. 10. 1958, daher im Buch von WERNERT 1957 noch nicht erwähnt). Er stammt aus dem roten sandigen „Lehm“ über dem roten Vogesensand der Schiltigheimer Terrasse. Die Schotter dieser Terrasse könnten nach WERnERT rißzeitliches Alter haben. In und auf diesem fluviatilen Lehm lagen die im Profil am höchsten gef undenen Backenzähne ( $\mathrm{M}_{1}$ und $\left.\mathrm{mm}_{2}\right)$ von Palaeoloxodon antiquus $\mathrm{F}_{\mathrm{ALC}}$. „où ses molares présentent un caractère évolutif très net".

Ein sehr viel schlechter erhaltener Unterkieferrest eines wesentlich jugendlicheren Riesenhirsches stammt von der "Langen Westwand der Lößgrube Michel“ aus dem Jahre 1938. Er lag direkt über der Moräne an einer Stelle, an der diese von Löß überdeckt war. Nach dem schlechten, aber kennzeichnenden Erhaltungszustand gehört dieses fossile Stück in das Lager der untersten Elefantenzähne und Nashornreste. Erhalten ist lediglich der $\mathrm{M}_{3}$ der rechten Kieferseite zusammen mit einigen Knochenresten. Der Vergleich mit anderen dritten Unterkiefermolaren zeigt, daß der besser erhaltene Unterkiefer sich durch besonders kräftige Zähne (und auch Bau des Knochens) auszeichnet. Der schlecht erhaltene Unterkiefer paßt in Zahn und Knochen weit besser in die Variation der übrigen Vergleichsstücke (Tabelle 3).

Tabelle 3

Maße der Unterkiefer von verschiedenen Riesenhirschen

\begin{tabular}{lccc}
\hline Maße d. $\mathrm{M}_{3}$ & Länge & $\begin{array}{c}\text { Größte Dicke des } \\
\text { mittleren Pfeilers }\end{array}$ & Höhe \\
\hline Murg, besser erhaltenes Fragment & 44 & $21-24$ & 20 \\
Murg, schlechter erhaltenes Fragment & 39 & 23,5 & 22,5 \\
Bad Tatzmannsdorf, Alleröd b. Wien & 38,5 & $19-21$ & $22-27$ \\
Klein Besen Mittenwalde & 41 & 20 & 18,2 \\
Nieder Lehme (Berlin) & 38 & 22 & -
\end{tabular}

Der zweite Riesenhirschunterkiefer ist, soweit es sich bei dem schlechten Erhaltungszustand erkennen läßt, ausgesprochen grazil, und der Rechen-Quotient: Höhe durch Dicke ist viel kleiner als bei den aus jüngeren Schichten stammenden Kiefern mit gleich großen Molaren.

In Murg fanden sich also dicht über dem Torfmoor ein sehr schmaler Kiefer und aus den kälteren und jüngeren Schichten ein ganz besonders kräftiges Dentale von besonderer Dicke.

Die Elef a nten. Von besonderer Bedeutung sind die Elefantenreste von Murg. Zur Zeit liegen 8 verschieden gut erhaltene Backenzähne vor. Ihre wichtigen Meßzahlen 


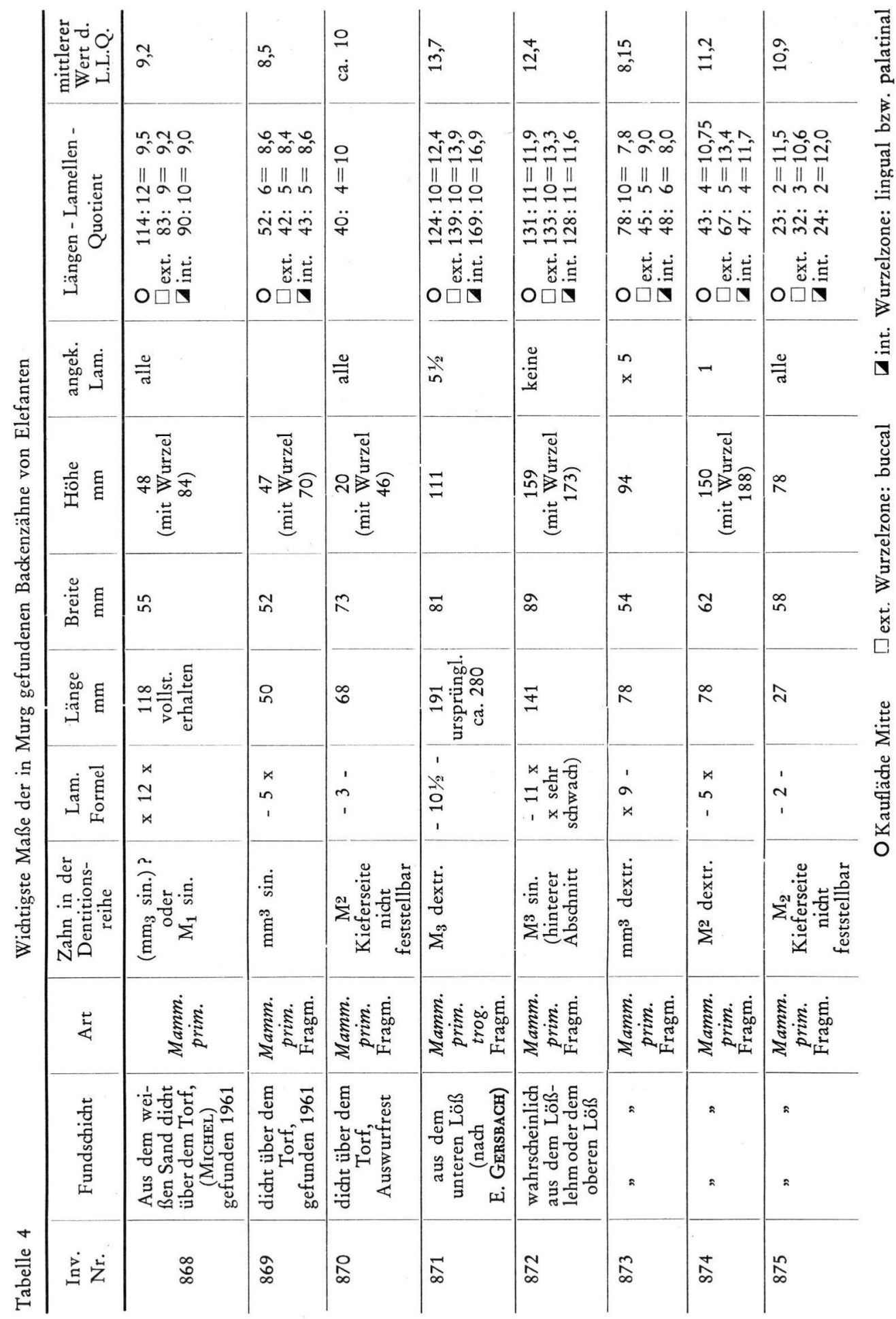


sind auf Tabelle 4 zusammengestellt. Zur Errechnung eines vergleichbaren Längen-Lamellen-Quotienten (L.L.Q.) werden mehrere Werte benötigt. Zunächst der L.L.Q. der Kauflächenmitte und weiter die lingualen (internen) und buccalen (externen) Werte der Wurzelbereiche. Nach diesen Zahlen läßt sich unter Berücksichtigung der Abkauungstiefe ein nicht mehr erhaltener L.L.Q., den der noch nicht abgekaute Zahn hatte, ziemlich genau abschätzen. Der mittlere L.L.Q. eines Molaren errechnet sich dann aus:

L.L.Q. des nicht angekauten Zahnes + mittl. Wurzelwert der lingualen und buccalen Seite

2

Damit ist eine Zahl errechnet, die vergleichbare Werte gibt und nicht anhängig ist vom Abkauungsgrad, der Krümmung der Molaren und dem Divergieren und Konvergieren der Lamellen von der Kaufläche zur Wurzel. Auf Abb. 3 sind die so errechneten mittleren Längen-Lamellen-Quotienten der Elefanten-Backenzähne von Murg eingetragen. Es zeigt sich, daß inner- und oberhalb des mächtigen Lehms (auf Abb. 3 zwischen 5 und $7 \mathrm{~m}$ Tiefe) Mammonteus primigenius auftritt, im Löß zwischen diesem Lehm und dem Torf eine Elefantenart, deren L.L.Q. hoch genug liegt, daß man von einem $M$. primigenius/trogontherii PoHL. sprechen kann. Auch andere Merkmale bestätigen diese Zugliederung. Dicht über dem Torf tritt dann wieder $M$. primigenius auf.

Diese Abfolge: In der Warmzeit Mammut, in der darauf folgenden Kaltzeit die Übergangsform $M$. primigenius/trogontherii und erst später wieder das eigentliche Mammut, konnte bei verschiedenen horizontiert gesammelten Fundserien beobachtet werden. Hiernach ergibt sich eine Möglichkeit der Parallelisierung zu Achenheim: Es entspricht der

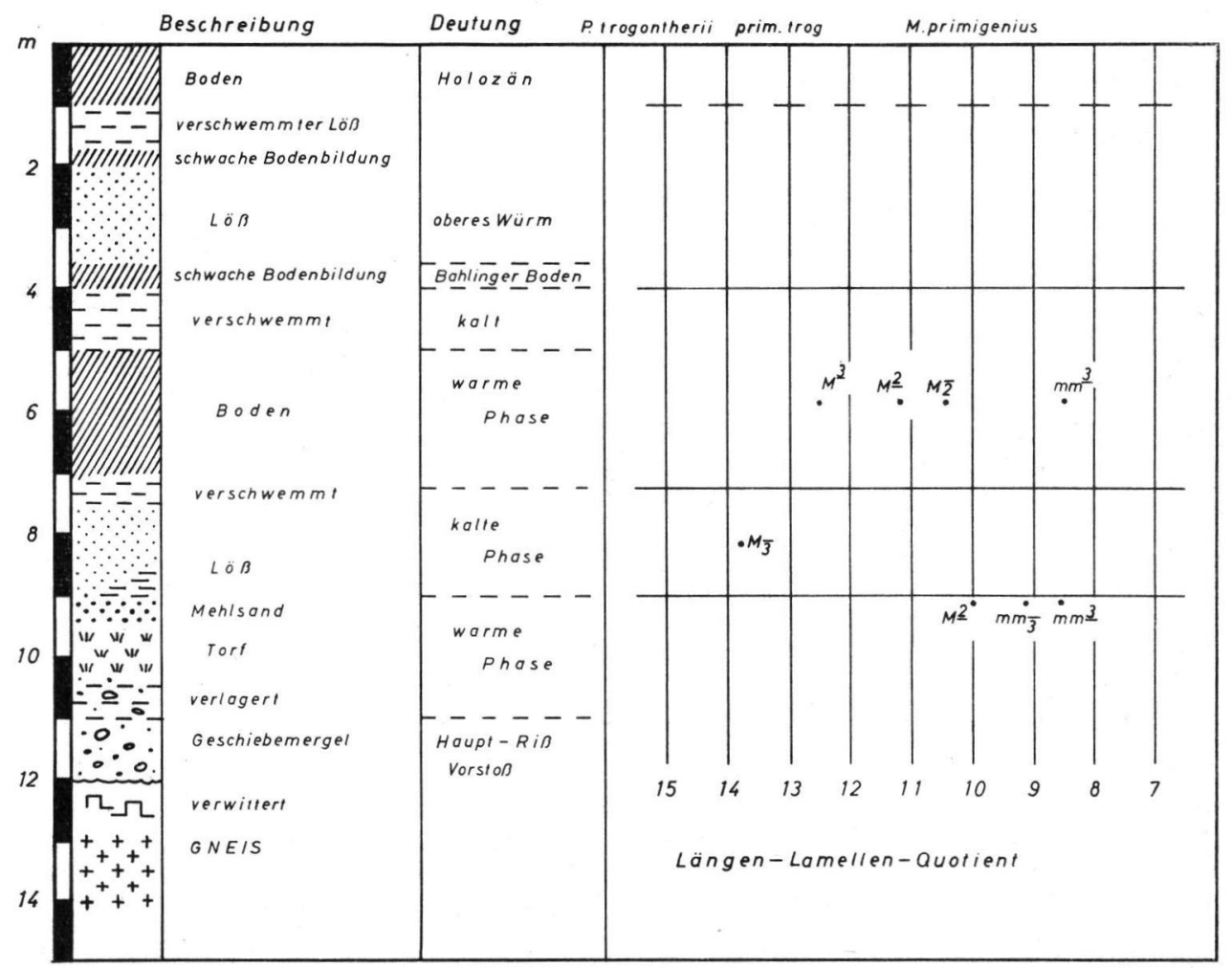

Abb. 3. Das Profil von Murg und die verschiedenen dort horizontiert gesammelten Elefantenarten. 
Lehm, der den Löß ancien moyen von Achenheim nach oben abschließt, zeitlich ungefähr dem Torf von Murg. Sein Klima wird von Wernert nach der Fauna bestimmt. Es ist gemäßigt, es gibt offene Wälder. Der Löß ancien supérieur entspricht im Murger Profil dem unteren Löß. Die Böden, die diese Schichten nach oben abdecken, entsprechen einander. WERNERT denkt an eine offene Wald- und Steppenvegetation (Taiga). Erst der darüber folgende Löß des Profils von Murg entspricht dem Loess récent von Achenheim.

$\mathrm{U} r$ : Von geringer stratigraphischer Bedeutung scheint der Nachweis von Bos primigenius BoJ. im unteren Abschnitt des Lößprofils dicht über dem Torf zu sein. Nach KunNSCHNYDER (1947) erscheint der Ur als asiatischer Einwanderer zwar erst im letzten Interglazial im Schweizer Lebensraum. WERNERT (1957) gibt ihn jedoch aus Schichten an, die bestimmt älter sind. Ferner wurde er in den Waldelefanten-Schottern von Steinheim/Murr bereits nachgewiesen.

Wollhaariges $\mathrm{Nashorn}$. An Nashornresten konnte ich im Heimatmuseum der Stadt Säckingen 2 Oberkiefermolaren und einige Zahnsplitter untersuchen. Die Oberkiefermolaren $\mathrm{M}^{3}$ und $\mathrm{M}^{1}$ oder ${ }^{2}$ hatten ein Etikett mit folgender Beschriftung: Kalvarienbergwand, Murg 1936, Lößgrube Michel, Südecke, zusammen mit Artefakten gefunden. Die Stücke lagen direkt auf dem Gneisriff, das hier von dem untersten Löß überlagert war. Die Zähne sind dunkel gefärbt und ähneln im Erhaltungszustand den Elefantenzähnen aus den untersten Lagen des L.össes dicht über den pollenführenden Schichten. Die Splitterstücke tragen die Bezeichnung: Murg 1941, Ostecke der Westwand, gefunden auf der Grubensohle, bei den beiden in der Wand steckenden Rollblöcken (Moräne) mit Kohlen. Die Bruchstücke stammen von einem linken Oberkiefermolaren und einige vielleicht auch von einem Unterkieferbackenzahn. Alle Stücke gehören zu Coelodonta antiquitatis (JAEG.), dem wollhaarigen Nashorn.

\section{Fauna, Altersbestimmung und Vergleich mit anderen Profilen}

Ein Teil der neu gefundenen, horizontiert gesammelten Fossilreste ist von Bedeutung für die Altersdatierung der einzelnen Schichten des Profils von Murg.

a. Einige Aussagekraft haben die Elefantenzähne. Acht horizontiert gesammelte Backenzähne erlauben zwar nicht die Überprüfung der Variationsbreite einzelner Merkmale, sie ermöglichen jedoch Vergleiche mit anderen Profilen. Am Ende der Zeit der Torfbildung lebte ein recht hoch entwickelter $M$. primigenius. Im überlagernden Löß findet sich dagegen die zumeist ältere Form $M$. primigenius-trogontherii, und im darüber folgenden Lehm und oberen Löß tritt nunmehr endgültig $M$. primigenius auf. Der Vergleich mit dem Profil von Achenheim, wo die gleiche Elefantenfolge auftritt, zeigt, daß die untere Primigenius-Form dicht über der Schicht mit Palaeoloxodon antiquus liegt.

b. Der Nachweis einer kleinen Bisonform direkt über dem Torflager bestätigt den Waldcharakter des Horizonts. In Achenheim stammt der Nachweis einer entsprechenden Waldform aus der Schicht des „Loess ancien atypique“ (Schicht 19) des „Loess ancien moyen", einem Horizont, der einem humushaltigen Lehm aufliegt, aus dem Palaeoloxodon antiquus nachgewiesen ist.

c. Die Unterkiefer der Riesenhirsche zeigen in verschiedenen Zeiten des Pleistozäns unterschiedliche Ausbildungen. Ein besonders kräftig entwickelter Unterkieferrest aus dem mittleren Löß von Murg hat Ähnlichkeit mit Unterkiefern von Phoeben bei Werder an der Havel, die vielleicht aus dem letzten Interglazial stammen. Aus der Entwicklungsreihe der Megaloceronten von Achenheim ergibt sich die beste Übereinstimmung mit einem Unterkieferast aus einem Lehm über der Schiltigheimer Terrasse. In diesem Lehm wurden 2 Backenzähne eines hochentwickelten Palaeoloxodon antiquus gefunden.

Alle drei Fossilarten machen die gleiche Aussage. Lediglich die Zone direkt über dem Geschiebemergel von Murg kann aus einem Zeitraum stammen, in dem Palaeoloxodon an- 
tiquus noch lebte. Wahrscheinlich war es sogar die der Torfbildung kurz vorangehende, vielleicht noch wärmere Zeitphase, die die letzten Waldelefanten sah. Damit ist die Torfschicht ungefähr gleichaltrig mit dem Travertin von Ehringsdorf und zwar eher mit dem oberen Travertin über dem Pariser (Guenther 1957/58).

\section{Menschliche Kulturen}

Die Ziegeleigrube von Murg hat Artefakte des Levallois (z. B. Schildkerne, Zotz 1951) und vielleicht auch des Acheuls und Moustériens geliefert. Die Kulturen werden z. Zt. von Dr. Egon Gersiach bearbeitet.

Auf menschlichen Aufenthalt weisen mehrere Feuerstellen hin, die während des Lößund Lehm-Abbaus verschiedentlich zu sehen waren. Funde fossiler Tierreste zusammen mit Artefakten erlauben den Schluß, daß wenigstens ein Teil der Knochen und Zähne die Reste menschlicher Jagdbeute sind. Die Elefantenzähne ermöglichen eine Aussage über das Alter der Tiere. Danach sind folgende Altersklassen vertreten:

$$
\begin{aligned}
& \text { 5-10 Jahre } 2 \text { Tiere 30-40 Jahre } 4 \text { Tiere. } \\
& \text { 20-30 Jahre } 2 \text { Tiere }
\end{aligned}
$$

Eine natürliche Sterbegemeinschaft besteht zum überwiegenden Teil aus Elefanten, deren Lebensalter über 40 Jahren liegt. Bei den Murger Zähnen handelt es sich um eine Auslese jüngerer Tiere, die sich am besten durch menschliche Jagd erklären läßt.

Von den palaeolithischen Feuerstellen wurden durch Wind und Niederschlagswasser feinste Kohleteilchen in weitem Umkreis um die eigentlichen Herdplätze ausgebreitet. Diese Kohlestückchen sind zum Teil so feinkörnig, daß eine Bestimmung nur unter dem Binokular möglich ist. Dazu fanden sich intensiv rot gefärbte Körnchen, wie sie entstehen, wenn Löß oder Lehm gebrannt werden. Abb. 2 zeigt in der Spalte „Fundplätze“, in welchen Horizonten nach diesen Untersuchungen die Herdstellen zu finden sind.

Die erste nachweisbare und nach den Beutestiicken nicht geringe Besiedlung findet sich bereits im Mehlsand und untersten Löß. Die größere Zahl der Herdstellen ist allerdings jünger, sie befindet sich im mächtigen Lehm.

\section{$\mathrm{Sch}$ riften}

Averdieck, F. R.: Das Interglazial von Fahrenkrug in Holstein. Ein Beitrag zur Frage des Buchenvorkommens im Jungpleistozän. - Eiszeitalter u. Gegenwart 13, 5-14, 1962.

FIRBas, F.: Über das Fagusvorkommen von Wasserburg am Inn (Oberbayern). - Veröff. geobot. Inst. Rübel in Zürich, Heft 33, 1958.

Guenther, E. W.: Feinstratigraphische Untersuchung einer Zwischenschicht und der Deckschichten der Travertine von Ehringsdorf. - Alt-Thüringen, Jahresschr. d. Museums f. Ur- und Frühgeschichte Thüringens, 3, S. 1-15, 1958. - - Funde von Ren und Riesenhirsch in nacheiszeitlichen Schichten aus der Nähe von Theresienhof bei Plön (Trentmoor und Brennacker). - Schriften naturw. Ver. Schleswig-Holstein 30, S. 79-91, Kiel 1960. - - Sedimentpetrographische Untersuchung von Lössen zur Gliederung des Eiszeitalters und zur Einordnung paläolithischer Kulturen. Teil I. - Böhlau Verlag, Köln, Graz 1961. - - Zur Bestimmung der Atlas-Wirbel einiger pleistozäner Cerviden und Boviden. - Mitt. geol. Staatsinst. Hamburg 31, S. 490-503, Hamburg 1962.

Hallik, R. \& Kubitzki, K.: Über die Vegetationsentwicklung des Weichsel-Interstadials aus Hamburg-Bahrenfeld. - Eiszeitalter u. Gegenwart 12, S. 92-98, 1961.

Hescheler, K. \& Kunn-Schnyder, E.: Die Tierwelt der praehistorischen Siedlungen der Schweiz. Aus Tschumi: Urgeschichte der Schweiz, S. 121-368, 1947.

LüDI, W.: Die Pflanzenwelt des Eiszeitalters im nördlichen Vorland der Schweizer Alpen. - Veröff. geobot. Institut Rübel in Zürich, Heft 27, 1953. - - Interglaziale Vegetation im schweizerischen Alpenvorland. - Veröff. geobot. Institut Rübel in Zürich, Heft 34, S. 99-107, 1958.

Penck, A. \& Brǘckner, E.: Die Alpen im Eiszeitalter. - Leipzig 1901 u. 1909. 
Pfannenstiel, M.: Die Vergletscherung des südlichen Schwarzwaldes während der Rißeiszeit. - Ber. naturf. Ges. Freiburg i. Br. 48, S. 231-272, Freiburg 1958. - - Das Rißglazial des Südschwarzwaldes. - Eiszeitalter u. Gegenwart 11, S. 228-229, OOhringen 1960. - - Glazialforschung im östlichen Schwarzwald. - Jb. d. Akad. d. Wiss. u. d. Lit. Mainz, 1961, S. 141, Mainz 1961.

Pfannenstiel, M. \& Rahm, G.: Die Vergletscherung des Wutachtales während der Rißeiszeit. Ber. naturf. Ges. Freiburg i. Br. 53, S. 5-61, Freiburg 1963.

REICH, Helga: Die Vegetationsentwicklung der Interglaziale von Großweil-Ohlstadt und Pfefferbichl im Bayerischen Alpenvorland. - Flora 140, S. 386-443, Jena 1953.

Reichelt, G.: Untersuchung zur Deutung von Schuttmassen des Südschwarzwaldes durch Schotteranalysen. - Beitr. z. naturk. Forsch. i. Südwestdeutschland 14, S. 32-42, Karlsruhe 1955. - - Zur Frage der Rißvereisung des Südschwarzwaldes. - Erdkunde 14, S. 53-5,8, Bonn 1960. - - Quartäre Erscheinungen im Hotzenwald zwischen Wehra und Alb. - Ber. naturf. Ges. Freiburg i. Br. 50, S. 57-127, Freiburg 1960.

Schmid, E.: Rißeiszeitliche Krotowine bei Murg am Hochrhein. - Mittbl. bad. geol. Landesanstalt Freiburg i. Br., S. 50, Freiburg 1949-50.

Schmidtgen, O., \& W WGNER, W.: Eine altpaläolithische Jagdstelle bei Wallertheim in Rheinhessen. - Notizbl. Ver. Erdkunde und hess. geol. L.A. Darmstadt (5) 11, S. 1-31, Darmstadt 1929.

Wernert, P.: Stratigraphie paléontologique et préhistorique des sediments quarternaires d'Alsace, Achenheim. - Mémoires du service de la carte géologique d'Alsace et de Lorraine 14, S. $1-355$, Strasbourg 1957.

Woldstedt, P.: Das Eiszeitalter, Grundlinien einer Geologie des Quartärs. Bd. 2, Stuttgart 1958.

ZINK, F.: Zur diluvialen Geschichte des Hochrheins und zur Altersstellung der paläolithischen Station Murg. - Mitt. Reichsstelle f. Bodenforschung, Zweigstelle Freiburg i. Br., H. 1, S. 1-51, Freiburg 1941.

Zotz, L.: Altsteinzeitkunde Mitteleuropas. - Stuttgart 1951.

Manuskr. eingeg. 1. 3. 1964.

Anschrift der Verf.: Prof. Dr. Ekke W. Guenther, 23 Kiel, Geol. Inst. d. Univers. Olshausenstr. 40/60. Prof. Dr. F. Tidelski, 23 Kiel, Clausewitzstr. 7. 